\title{
Imaging for suspected colorectal cancer in frail and elderly patients
}

\author{
L. Bacigalupo $\cdot$ F. Paparo
}

Received: 21 July 2013/Accepted: 26 July 2013/Published online: 9 August 2013

(C) Springer-Verlag Italia 2013

Medicine faces many challenges, among which that of the growing number of old and often frail patients needs to be particularly addressed. In our hospital, that serves one of the oldest population on earth (about $10 \%$ of the inhabitants of Genoa are over 80 years old) problems related to diagnosing and treating pathologies in this type of patients are part of our daily practice. Many old patients enter our hospital for gastrointestinal symptoms possibly related to colorectal cancer, often via the emergency room.

In these patients (in which it is frequently difficult to obtain a good clinical history), there is the need to make a diagnosis, preferably noninvasively, to plan appropriate treatment.

In colorectal cancer, computed tomography (CT) offers different modalities that allow to diagnose and stage correctly the disease. The most commonly used are two that require good bowel preparation:

- CT colonography requires distention of the colon with gas (room air or, preferably, carbon dioxide), usually without iodinated contrast media intravenously but often with orally administered iodinated contrast media (and/or barium) before the examination to properly "tag" residual feces and liquids in the colon $[1,2]$. It is possible to add intravenous contrast media to CT colonography before the examination in patients with high pretest likelihood of positive findings (colon cancer or significant extra-colic findings) [3]. Intravenous contrast media can also be added when required during the CT colonography itself after the first (usually supine) acquisition; but this requires the presence the

L. Bacigalupo $(\varangle) \cdot$ F. Paparo

Galliera Hospital, Mura delle Cappuccine 14, 16128 Genoa, Italy

e-mail: lorenzo.bacigalupo@galliera.it immediate evaluation of the radiologist during the CT examination, which can be difficult in a real clinical setting (because of the high workflow in the CT suite) and since often colorectal cancer is depicted only after extensive review of the CT images. In CT colonography performed after tagging, intravenous contrast media may, in our experience, make more difficult the assessment/depiction of enhancing bowel wall lesions located in segments with superimposable hyperdensity of the adjacent bowel content. In our opinion, CT colonography should be mainly reserved for old outpatients in good condition, in whom significant colon pathology must be ruled out and there is a low pretest likelihood of significant findings. Hospitalized old and frail patients may have problems in completing the full CT colonography preparation and even in undergoing the CT scan itself with the two required positions of the patient (supine and prone).

- Water enema CT is performed after distension of the colon with water and, always, with intravenous iodinated contrast media [4]. This technique can be modified to obtain distention of the small bowel with oral administration of a negative contrast medium such as a polyethylene glycol electrolyte solution [5]. Water enema CT is mainly used in our hospital in those old and frail patients with a high pretest likelihood of colon cancer, based on clinical suspicion. The use of iodinated contrast media intravenously allows to easily and completely assess the whole abdomen, particularly the liver (with at least portal and venous phases to differentiate simple liver cysts from colorectal cancer metastases) $[6,7]$. The chest can also be fully evaluated during water enema CT mainly for metastases from colorectal cancer (providing an important baseline CT study for follow-up) and also for cardiovascular 

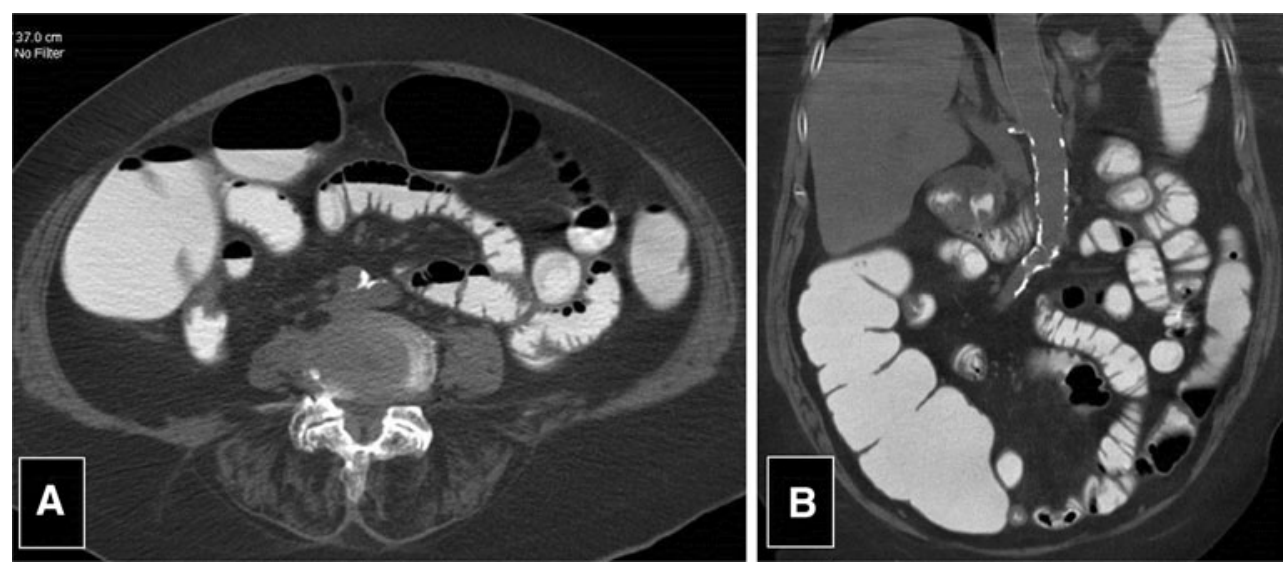

Fig. 1 a and b A 80-year-old lady with symptoms of partial bowel occlusion. We can see the colon well opacified by opaque enema (iodinated contrast media diluted in water) and distended by minimal air insufflation. In this old patient, due to incontinence of the ileocecal

surgical risk assessment (particularly atheromatous disease, coronary plaques and previous silent myocardial infarcts) with modern MDCT machines [8-10]. The main caveat, if water enema CT is planned, is to ascertain that the patient has a normal renal function.

It is also possible to assess the colon, and the abdomen, of old patients with other two types of CT scan that can be performed with minimal preparation of the bowel:

- Minimal preparation $C T$ of the colon with tagging of the bowel content with iodinated contrast media (with or without iodinated contrast media intravenously) such as the technique used in the article by Saunders and colleagues [11]. This technique can be improved with minimal distention of the colon with gas [12]. This type of CT scan is, in our experience, the test of choice in old and frail patients unable to complete bowel cleansing and particularly in those that have impaired renal function. Minimal distention of the colon with air does not require dedicated equipment and should be performed whenever it is feasible. It is important to remember that aspiration of iodinated contrast media can cause pulmonary edema, and dysphagia (not uncommon in elderly patients) has to be ruled out before administration of oral contrast [13].

- CT opaque enema is done with an enema composed of water and diluted iodinated contrast media, and this type of study can be also done after "tagging" of the bowel with the use of oral iodinated contrast media and allows to assess both the small and large bowel (Fig. 1). In our clinical practice, in some old and frail patients who were referred for CT colonography, this type of examination is performed when the patient appears unfit to tolerate the distention with gas and/or the prone/ lateral decubitus. This type of CT study may be valve, a good evaluation of the small bowel was also obtained. CT was performed without the use of intravenous iodinated contrast media

particularly useful in patients with colorectal fistulas and/or perforations that can be observed as complications of colorectal cancer.

These four different CT examinations make it possible to obtain three-dimensional images of the colon and offer at the same time, an evaluation of the abdomen that can be further improved using intravenous iodinated contrast media when possible and/or needed. In the majority of patients, significant colorectal pathology can be excluded or confirmed with CT. Optical colonoscopy can be then reserved for patients with positive and/or doubtful findings at $\mathrm{CT}$ and patients needing biopsy and/or other procedures (stent placement for instance). In the future, we can therefore expect that the role of CT for the diagnosis of colon disease will expand, particularly in old patients.

It has to be stressed that in old patients, the risk of radiation from CT is totally negligible, instead the risk of perforation during optical colonoscopy and of incomplete optical colonoscopy have to be considered. The main risk of CT for old patients is contrast-induced nephropathy; therefore, intravenous iodinated contrast media have to be limited to patients with an adequate creatinine clearance and multiple contrast-enhanced CT should be avoided. It is also important to emphasize the fact that that $\mathrm{CT}$ can always be easily extended (without or with intravenous iodinated contrast media) to the assessment of the chest and/or head-neck allowing a "one stop" whole body diagnostic procedure.

The article of Saunders and colleagues supports the use of $\mathrm{CT}$ as a problem solver in old and frail patients, making it possible to diagnose colonic and extra-colonic pathology, focusing on the clinical outcome based on life expectancy. In each case, the different possible CT examinations of the colon should be considered based on the available evidence 
in order to tailor the CT procedure to each patient. The only way to achieve this is with a close collaboration between surgeons and radiologists.

Conflict of interest None.

\section{References}

1. Laghi A, Rengo M, Graser A, Iafrate FJ (2013) Current status on performance of CT colonography and clinical indications. Eur J Radiol 82:1192-1200

2. Mang T, Schima W (2013) CT colonography—a guide for clinical practice. Thieme, Stuttgart. ISBN 3131506210

3. Neri E, Halligan S, Hellström M et al (2013) The second ESGAR consensus statement on CT colonography. Eur Radiol 23:720-729

4. Soyer P, Hamzi L, Sirol M et al (2012) Colon cancer: comprehensive evaluation with 64-section CT colonography using water enema as intraluminal contrast agent-a pictorial review. Clin Imaging 36:113-125

5. Paparo F, Garlaschi A, Biscaldi E, Bacigalupo L, Cevasco L, Rollandi GA (2013) Computed tomography of the bowel: a prospective comparison study between four techniques. Eur $\mathbf{J}$ Radiol 82:e1-e10

6. Motosugi U, Ichikawa T, Nakajima $\mathrm{H}$ et al (2009) Imaging of small hepatic metastases of colorectal carcinoma: how to use superparamagnetic iron oxide-enhanced magnetic resonance imaging in the multidetector-row computed tomography age? J Comput Assist Tomogr 33:266-272

7. Schima W, Kölblinger C, Ba-Ssalamah A (2012) Non-invasive diagnosis of focal liver lesions: an individualized approach. Cancer Imaging 12:365-372

8. Christoffersen MW, Bulut O, Jess P (2010) The diagnostic value of indeterminate lung lesions on staging chest computed tomographies in patients with colorectal cancer. Dan Med Bull 57:A4093

9. de Malherbe M, Duhamel A, Tacelli N et al (2012) Ultrafast imaging of the entire chest without ECG synchronisation or betablockade: to what extent can we analyse the coronary arteries? Insights Imaging 3:73-79

10. Shriki JE, Shinbane J, Lee $C$ et al (2012) Incidental myocardial infarct on conventional nongated CT: a review of the spectrum of findings with gated CT and cardiac MRI correlation. AJR Am J Roentgenol 198:496-504

11. Saunders JH, Miskovic D, Bowman C, Panto P, Menon A (2013) Colorectal cancer is reliably excluded in the frail and elderly population by minimal preparation CT. Tech Coloproctol. doi:10. 1007/s10151-013-1045-4

12. Slater A, North M, Hart M, Ferrett C (2012) Gas insufflation of minimal preparation $\mathrm{CT}$ of the colon reduces false-positives. Br J Radiol 85:346-350

13. Trulzsch DV, Penmetsa A, Karim A, Evans DA (1992) Gastrografin-induced aspiration pneumonia: a lethal complication of computed tomography. South Med J 85:1255-1256 